\title{
Variability of EUV-spectra from the quiet upper solar atmosphere: Intensity and Doppler shift
}

\author{
A. Brković ${ }^{1,3}$, H. Peter ${ }^{1}$, and S. K. Solanki ${ }^{2}$ \\ ${ }^{1}$ Kiepenheuer-Institut für Sonnenphysik, Schöneckstr. 6, 79104 Freiburg, Germany \\ 2 Max-Planck-Institut für Aeronomie, Max-Planck-Str. 2, 37191 Katlenburg-Lindau, Germany \\ ${ }^{3}$ Institute of Astronomy, ETH-Zentrum, 8092 Zürich, Switzerland
}

Received 15 May 2002 / Accepted 11 March 2003

\begin{abstract}
We have studied SUMER and CDS time series of spectra and images of quiet-Sun regions at the solar disc centre. The data contain ultraviolet emission lines sampling temperatures of the chromosphere, transition region and corona. We find a high correlation between average net Doppler shifts and relative brightness variabilities of the studied lines (correlation coefficient of 0.92), suggesting a connection between the two quantities. The anti-correlation between differential emission measures and relative brightness variabilities is weaker (correlation coefficient of -0.78). We discuss the observed relationships on the basis of differential emission measures and linear wave calculations.
\end{abstract}

Key words. Sun: chromosphere - Sun: corona - Sun: transition region - Sun: UV radiation

\section{Introduction}

Recent EUV observations have revealed that transition region lines are on average redshifted, while the coronal lines are blueshifted (Peter \& Judge 1999; Teriaca et al. 1999; and references therein). Models proposed to explain these shifts include siphon flows through loops, explosive events, waves due to nano-flares or return of spicular material (Antiochos 1984; McClymont \& Craig 1987; Mariska 1988; Hansteen 1993; Spadaro et al. 1996; for a discussion see Peter \& Judge 1999). Early studies of the correlation between Doppler shifts and line intensities in the quiet Sun gave inconsistent results. Athay et al. (1983) and Dere et al. (1984) found no correlation between intensity and velocity in the data covering the C IV $1548 \AA$ transition region line. Gebbie et al. (1981) analysed spectra of the C II $1336 \AA$, Si IV $1393 \AA$ and C IV $1548 \AA$ lines and found that redshifted regions were correlated with regions of bright network emission and blueshifted regions tend to be associated with darker areas. More recently, Stucki et al. (2000) and Hansteen et al. (2000) both obtained a positive correlation between network emission and redshift of transition region lines in the quiet Sun. In addition, Stucki et al. (2000) also showed that in coronal holes, the sign of the correlation is reversed. Such a correlation supports the model of Hansteen (1993) who proposed that nano-flares occurring at the top of coronal loops generate MHD waves that propagate downward along the magnetic fields towards and through the transition

Send offprint requests to: A. Brković, e-mail: alen@kis.uni-freiburg.de region in their footpoints, which lie in the network. The net redshifts in TR spectral lines are a result of the correlation between the intensity and velocity that occurs in downward propagating acoustic waves.

Studies of line intensity variability (e.g., Rabin \& Dowdy 1992; Harrison 1997; Krucker et al. 1997; Brković et al. 2000) as well as of line shifts are numerously represented in the literature, the latter both on theoretical and observational basis (see above). Although both quantities have been related to other parameters, e.g., line formation temperature, intensity (at a given spatial location) and line width, the two quantities have never so far, to our knowledge, been compared with each other for a group of spectral lines. This work tries to establish the connection between spatial averages of relative changes in line intensities and of net Doppler shifts. The former are observed in the quiet Sun using time series of spectra recorded by SUMER and movies obtained with CDS. The latter are taken from the literature. In addition we analyse the relationship between differential emission measures and relative intensity variabilities.

After the description of the observations in Sect. 2 we describe in Sect. 3 our results concerning the time variability of line intensities and Doppler shifts and the relation to the differential emission measure and the intensity distribution. These results are discussed in Sect. 4 with respect to selection effects due to the emission measure distribution, and a simple model to understand the close relation between Doppler shift and intensity fluctuations in terms of linear waves are described. Before concluding the paper some remarks are made on the structure of the transition region. 
Table 1. Observed lines. Asterisks denote ions observed by Peter \& Judge (1999) or Teriaca et al. (1999). Temperatures at peak of the ion's relative abundance $T_{\mathrm{e}}$ follow Landini \& Monsignori Fossi (1990).

\begin{tabular}{|c|c|c|c|c|}
\hline Line $(\AA$ & & $\log \left(T_{\mathrm{e}} / \mathrm{K}\right)$ & Line $(\AA)$ & $\log \left(T_{\mathrm{e}} / \mathrm{K}\right)$ \\
\hline $\operatorname{Mg}$ IX & 368.1 & 6.00 & $\mathrm{OI}^{*} \quad 1152.1$ & 4.18 \\
\hline $\mathrm{He}^{*}$ & 584.3 & 4.50 & $\mathrm{C}_{\text {III }}{ }^{*} 1175.7$ & 4.80 \\
\hline $\mathrm{O} v^{*}$ & 629.7 & 5.39 & $\begin{array}{ll}\mathrm{C}^{*} & 1267.7\end{array}$ & 4.18 \\
\hline $\mathrm{N}_{\text {III }}$ & 764.4 & 4.90 & $\mathrm{OI}^{*} \quad 1302.2$ & 4.18 \\
\hline $\mathrm{N}_{\text {IV }}$ & 765.2 & 5.20 & O I $^{*} \quad 1304.9$ & 4.18 \\
\hline Ne viII* & * 770.4 & 5.81 & $\mathrm{Si}_{\text {II }}^{*} 1309.3$ & 4.10 \\
\hline $\mathrm{S} \mathrm{VI}^{*}$ & 933.4 & 5.28 & $\mathrm{C}_{\text {III }^{*}} 1334.5$ & 4.60 \\
\hline $\mathrm{O} \mathrm{vI}^{*}$ & 1031.9 & 5.47 & & \\
\hline
\end{tabular}

\section{Observations}

For the evaluation of the variabilities in both intensity and Doppler shift as well as differential emission measures quiet regions at Sun centre have been observed using the Solar Ultraviolet Measurements of Emitted Radiation (SUMER) spectrometer (Wilhelm et al. 1995) and the Normal Incidence Spectrometer (NIS) of the Coronal Diagnostic Spectrometer (CDS, Harrison et al. 1995) onboard the SOHO spacecraft. A list of the lines analysed and temperatures of line formation are given in Table 1 . The Mg Ix $368.1 \AA$ line was observed only with CDS, He I $584.3 \AA$ and O v $629.7 \AA$ were observed with both CDS and SUMER; all other lines were observed only with SUMER. These lines cover chromospheric, transition region and coronal temperatures.

SUMER observed with detector B on 14 and 16 February 1997 (R. Thomas) and 25 February 1997 (D. Gigas) using the $1^{\prime \prime} \times 300^{\prime \prime}$ slit \#2 and on 22, 23 and 25 April 1997 (I. Rüedi) using $120^{\prime \prime}$ long slits \#3 ( $1^{\prime \prime}$ wide) and \#6 (0.3" wide). The pixel size was $1^{\prime \prime} \times 1^{\prime \prime}$, except for the slit \#6 where it was $0.3^{\prime \prime} \times 1^{\prime \prime}$. The SUMER slit was kept at a fixed location on the solar surface by compensating for solar rotation. Several instrumental corrections have been applied to the data before the analysis. For the flat-field correction we used flat-field images taken on 27 February 1997 and on 24 April 1997. The pin cushion distortion of the image and the inclination of the spectral lines with respect to the detector columns were removed. The effects of the dead-time and gain-depletion of the detector were almost negligible, but the corrections due to these effects have been applied anyway. In the next step we fit the line profiles, except for the $\mathrm{N}_{\text {III }} 764.4 \AA$ and $\mathrm{C}_{\text {III }} 1175.7 \AA$ lines, at each spatial position and for each time step. For all lines least-squares fits of a single Gaussian plus a linear background turned out to be sufficient. The fitting procedure failed to give reliable fits for $\mathrm{N}$ III due to a low signal-to-noise ratio and for $\mathrm{C}$ III which is strongly blended (actually it is a multiplet of six $\mathrm{C}$ III lines). Since we were interested only in the variations of the total intensity formed at a particular temperature we spectrally summed over the line profile after subtracting for the continuua determined from the N IV 765.2 $\AA$ and He I $584.3 \AA$ (recorded in 2nd order) lines, respectively.

CDS/NIS was employed in its movie mode, i.e., with a $90^{\prime \prime} \times 240^{\prime \prime}$ slit. In this mode a filtergram covering a part of the solar surface corresponding to the slit size is produced simultaneously at each wavelength. Due to the overlap of the images from neighbouring wavelengths spectral information within each spectral line is lost. After correction for solar rotation, performed on the ground, each pixel follows the same point on the solar surface during the whole time series. To the actual exposure time an overhead of four or five seconds per frame must be added (the overhead is mainly accrued by reading out the CCD and preparing it for the next exposure). Due to telemetry constraints somewhat less than half of the data along the slit were read out and consequently only a smaller area of the solar disc is covered. The correction for solar rotation further reduces the size of the field of view, which finally is $70^{\prime \prime} \times 109^{\prime \prime}(42 \times 65)$ pixels, with a pixel size of $1.68^{\prime \prime} \times 1.68^{\prime \prime}$.

For more information about observations related to the variability see also Brković et al. (2002) and for observations which provided absolute shifts see Peter \& Judge (1999) and Teriaca et al. (1999).

\section{Results}

\subsection{Intensity and Doppler shift fluctuations}

The time variability $\delta I$ is described by the RMS variation of the intensity during the time series. The (average) intensity is the average over the whole duration of the observations. The relative variability $\delta I / I$ is defined as the ratio of the RMS to the intensity. These three parameters were determined for each spatial pixel for the spectral lines of interest. Finally, averages over all spatial locations were formed for each line. A similar procedure was employed to determine the RMS fluctuations of the (relative) Doppler shifts.

Figure 1a shows the Doppler shift variability of each spectral line as a function of its temperature of formation. The noise in the variability is found as the average of line position errors determined from fits, over the period of observations and has been removed. Negative values in the plot reflect the fact that the lines formed at low temperatures $(\log T<4.2)$ show very small RMS fluctuations of the line position, which are smaller than the noise introduced by the fitting errors, i.e., for these lines we do not detect a solar RMS fluctuation in Doppler shift. In Figs. 1b,c we plot the relative intensity variability and mean Doppler shift as a function of its temperature of formation. Note that the variabilities obtained by CDS, denoted by squares in Fig. 1b, have been corrected in order to make them comparable to the SUMER results (cf. Brković et al. 2002). The error bars denote standard deviations. The arrow attached to the symbol representing Mg IX indicates that the measured variability of this line is an upper limit, due to noise.

Since measuring absolute shifts was not one of the original aims of these observations they are not well suited to obtaining reliable values of the mean shift. We have therefore preferred to use the values published by Peter \& Judge (1999) and by Teriaca et al. (1999). We face the problem that these authors analysed some spectral lines not present in our sample and vice versa. There are twelve common ions, designated by asterisks in the Table 1, for which measured values of both the relative variability and the Doppler shift are available. In order to preserve the advantages of both investigations (the data of 

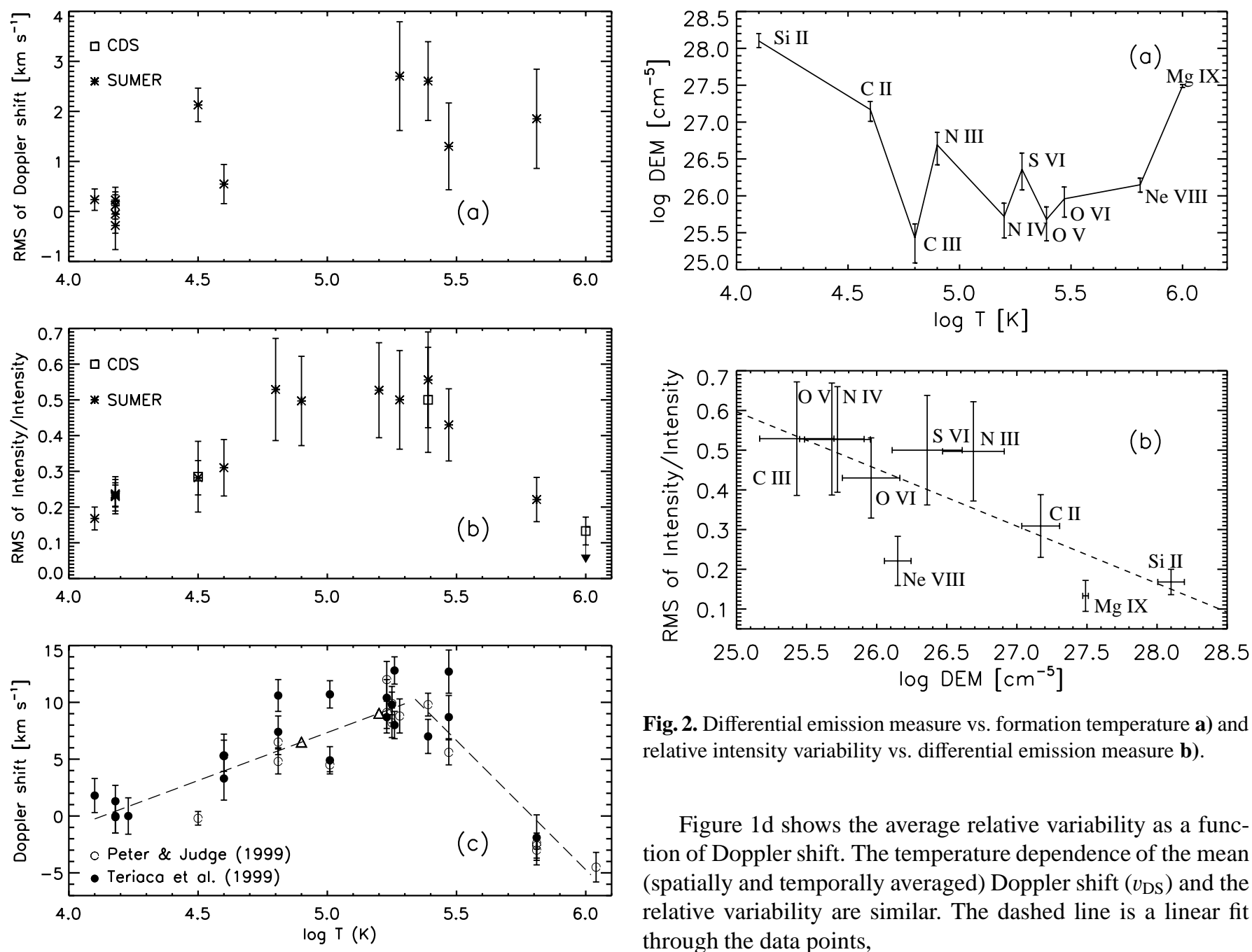

Fig. 2. Differential emission measure vs. formation temperature a) and relative intensity variability vs. differential emission measure b).

Figure 1d shows the average relative variability as a function of Doppler shift. The temperature dependence of the mean (spatially and temporally averaged) Doppler shift ( $\left.v_{\mathrm{DS}}\right)$ and the relative variability are similar. The dashed line is a linear fit through the data points,

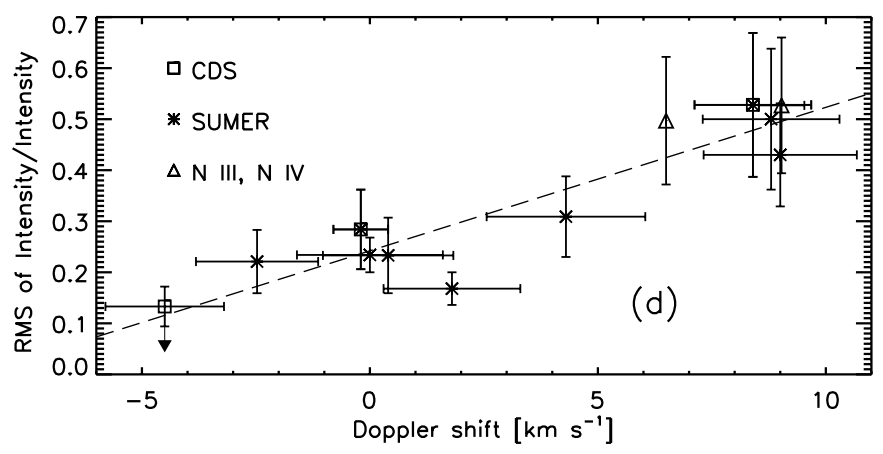

$\delta I / I=0.24( \pm 0.02)+0.029( \pm 0.004) v_{\mathrm{DS}}$.

The correlation coefficient between the relative variability and Doppler shift is 0.92 . According to probability calculus this extremely tight relationship has a probability below $10^{-3}$ of being due to chance, suggesting a physical connection between these two quantities. A proposal for this connection is presented in Sect. 4.2.

\subsection{Differential emission measure}

Fig. 1. Doppler shift variability a), relative intensity variability b) and mean Doppler shift c) vs. formation temperature and relative variability vs. mean Doppler shift d).

Teriaca et al. 1999 contain more lines, while only Peter \& Judge 1999 include a truly coronal line in their sample) we have combined the mean velocities from both and fit them with 2 straight lines, one for $\log T<5.3$, the other for $\log T \geq 5.3$ (dashed lines in Fig. 1c). From the fit for $\log T<5.3$ we obtained Doppler shifts for $\mathrm{N}_{\text {III }}$ and $\mathrm{N}_{\text {IV }}$ (triangles in Fig. 1c), therefore we did not estimate errors for these lines. The estimated Doppler shift of $9.2 \mathrm{~km} \mathrm{~s}^{-1}$ for $\mathrm{N}_{\mathrm{IV}} 765.2 \AA$ agrees very well with the value of $9 \mathrm{~km} \mathrm{~s}^{-1}$ published by Brekke et al. (1997).

Comparing Fig. 1b and the behaviour of the differential emission measure (DEM) up to one million $\mathrm{K}$ (the maximum temperature of our observations) we notice that the relative variabilities and DEMs are anti-correlated. Recall that DEM as a function of $T$ decreases from $10^{4} \mathrm{~K}$ towards higher temperatures. Near $3 \times 10^{5} \mathrm{~K}$ it reaches its minimum, then it increases until $1 \times 10^{6} \mathrm{~K}$ and finally it decreasess again. For the quantification of the relation of the DEM and the RMS variations of the intensity we calculated DEMs using the CHIANTI package (Dere et al. 1997). Of course, for the optically thick He I line this does not make sense because of uncertainty in the temperature of formation, so we skipped it. The plot of log DEM as a function of $\log T$ is shown in Fig. 2a. Our results are in a fair agreement with the standard DEM curve (cf. Mariska 1992). 
In the next step we directly compare log DEMs and relative variabilities (Fig. 2b). The dashed line is a linear fit through the data points. A simple comparison by eye of Figs. $1 \mathrm{~d}$ and $2 \mathrm{~b}$ shows that for our data the relation of the RMS fluctuations to the Doppler shifts is much closer than that of the RMS fluctuations to the DEM. This is quantified by the correlation coefficients, which are 0.92 and -0.78 , respectively. This might imply that relative variabilities are related more strongly to Doppler shifts than to differential emission measures. However more data are needed to draw a final conclusion.

\subsection{Intensity distributions}

Another interesting point which deserves to be mentioned is the result presented by Wilhelm et al. (1998), who analysed full Sun and Sun centre observations during the minimum of solar activity in 1996. They found that the logarithms of line radiances are normally distributed. Their plot of the widths of the distributions (their Fig. 9) is qualitatively similar to our Fig. 1 b. If one considers the distribution of intensities to be largely due to temporal fluctuations, one should expect the width of the intensity distribution to be related to the RMS fluctuations. This argument still holds when considering spatial variations and assuming that the different locations on the Sun are independent. In this sense the RMS fluctuations (Fig. 1b) and the widths of the intensity distributions (Wilhelm et al. 1998, Fig. 9) are different ways to look at the same problem under the assumption outlined above (see also Pauluhn et al. 2000).

\section{Discussion}

In the previous section we showed that relative variabilities of intensity are highly correlated to Doppler shifts and modestly anti-correlated to differential emission measures. We proceed with two simple, but different interpretations of these results, before discussing implications for the transition region structure.

\subsection{Relation to the differential emission measure}

Even though the anti-correlation between the differential emission measure (DEM) and the RMS variations of the intensity is not very high (Sect. 3.2) we will outline a scenario for this anti-correlation.

Using the definition of the DEM (e.g. Mariska 1992) for constant pressure $p \propto n T$, with the (electron) number density $n$, the DEM is given by

DEM $:=n^{2} \frac{\mathrm{d} z}{\mathrm{~d} \log T} \propto p^{2} \frac{1}{T} \frac{\mathrm{d} z}{\mathrm{~d} T} \propto\left(\frac{\mathrm{d} T^{2}}{\mathrm{~d} z}\right)^{-1}$.

This implies that the DEM is smallest where the temperature gradient is largest and vice versa. Taking, for the sake of argument an anti-correlation of DEM and RMS intensity fluctuations for granted this means that the latter are largest where the temperature gradient is steepest.

This leads to a selection effect which may give rise to a correlation between intensity fluctuations and DEM (cf. Fig. 2b).
When the DEM gets smaller, the amount of emitting material gets smaller and thus there is less averaging along the line of sight (assuming that the individual structures do not become correspondingly finer). Thus one would expect the strongest fluctuations where the DEM is smallest. Conversely, at temperatures where the DEM is large (e.g. large scale height at coronal temperatures) there is a more effective averaging along the line of sight. Additionally, there may be more structures along the line of sight, causing the observed fluctuations to decrease with $T$, while the local fluctuations increase.

\subsection{Relation to compressible fluctuations}

Here we propose that the process leading to the tight relation of the intensity RMS variations and the net Doppler shifts as shown in Fig. 1d is based on compressible fluctuations. This was inspired by sound waves, i.e., longitudinal compressions, which lead also to net Doppler shifts: towards the red if the wave is propagating away from the observer, towards the blue in the opposite case. This behaviour can be shown in simple terms analytically for optically thin lines formed in an isothermal constant-pressure atmosphere. Investigating this effect Hansteen (1993) numerically modeled coronal loops including sound waves propagating along the loops in order to understand the transition region line shifts (solving the full 1D problem along the loop).

Even though the real Sun is much more complicated, we use the simple ansatz that compressible fluctuations cause a sinusoidal variation of the intensity at line centre, $\widetilde{I}$, at a given location in the atmosphere, where the respective line of interest is formed,

$\widetilde{I}(t)=\widehat{I} \sin (\omega t)+I_{0}$,

where $I_{0}$ can be considered as the mean line-centre intensity. Accordingly the velocity, $\widetilde{v}$, varies as

$\widetilde{v}(t)=\widehat{v} \sin (\omega t+\varphi)$.

Here $\widehat{I}$ and $\widehat{v}$ denote the amplitude of the intensity and velocity fluctuations, the wave frequency is given by $\omega=2 \pi / \tau$, with $\tau$ being the period of the wave, and $t$ denotes the time.

The phase lag, $\varphi$, between the intensity and the velocity fluctuations is 0 for a pure upward moving sound wave and $\pi$ for a downward propagating sound wave. Because of this the upward moving sound wave causes net blueshifts: for $\varphi=0$ the intensity $\widetilde{I}$ is at its maximum when the gas is moving upwards, $\widetilde{v}>0$, and the intensity is lowest when the gas is moving downwards, $\widetilde{v}<0$. A net blueshift results by averaging over one period $\tau$. Similarly this gives a redshift for a downward propagating wave with $\varphi=\pi$.

With this simple ansatz (3) and (4) it is implicitly assumed that the atmosphere is "piecewise" isothermal, i.e., over the height of formation of the respective line the temperature remains constant. However, the atmosphere is not as simple as that and for a correct model one would have to investigate also effects of the atmospheric structure as well as of heat conduction and radiation; especially the time scales involved in the latter processes. Waves of any period can produce a net Doppler 
shift, even when not resolved by observations (Hansteen 1993). Therefore as a working hypothesis we assume that compressible fluctuations with periods shorter than currently resolvable by observations, both temporally and spatially, are leading to the observed net Doppler shifts.

Note, however, that in order to produce detectable velocity and intensity fluctuations (and not just cause lineshifts from unresolved motions, e.g. Wikstøl et al. 1997), the wavelength $\lambda$ should be at least of the order of the line formation length $L$. Otherwise different (non-coherent) wave packages might cancel each other. This puts a lower limit on the period, $\tau=\lambda / c_{\mathrm{s}}$, of the wave, i.e., $\tau \geq L / c_{\mathrm{s}}$, with $c_{\mathrm{s}}$ being the sound speed. For typical transition region values, $c_{\mathrm{s}}=50 \mathrm{~km} \mathrm{~s}^{-1}$ at $10^{5} \mathrm{~K}$, $L=20 \mathrm{~km}$ (e.g., Mariska 1992) this would put the lower limit of the wave period to $\tau \geq 0.4 \mathrm{~s}$.

Using the above ansatz (3) and (4) the line profile at time $t$ is then given by

$I_{v}(t)=\widetilde{I}(t) \exp \left[-\left(\frac{v-\widetilde{v}(t)}{\sigma}\right)^{2}\right]$,

with the (thermal) line width $\sigma$, i.e. the half line width at $1 / e$ of the peak intensity. Here the wavelength is given in velocity units and denoted by $v$.

As the fluctuations are assumed to be un-resolved in time, one has to average the spectrum over one (or more) wave periods, which is given by

$\left\langle I_{v}\right\rangle=\frac{1}{\tau} \int_{0}^{\tau} I_{v}(t) \mathrm{d} t$.

This is the theoretical spectrum that one has to compare to observations (after taking into account additional broadening due to, e.g., turbulence on the Sun and instrumental effects). Here we follow Hansteen (1993) and analyse the mean spectrum $\left\langle I_{v}\right\rangle$ in terms of its moments.

The zeroth moment of the line profile, $\int\left\langle I_{v}\right\rangle \mathrm{d} v$, is the (mean) total intensity, $I_{\text {tot }}$. By using expressions (3)-(6) and evaluating the integrals over wavelength and time this results in

$I_{\mathrm{tot}}=\int_{-\infty}^{+\infty}\left\langle I_{v}\right\rangle \mathrm{d} v=\sqrt{\pi} I_{0} \sigma$,

i.e., the total intensity is given by the mean line-centre intensity $I_{0}$ and the line width $\sigma$.

The line position, i.e., the Doppler shift, $v_{\mathrm{D}}$, is given by the first moment of the line profile:

$v_{\mathrm{D}}=\frac{1}{I_{\mathrm{tot}}} \int_{-\infty}^{+\infty}\left\langle I_{v}\right\rangle v \mathrm{~d} v=\frac{1}{2} \frac{\widehat{I}}{I_{0}} \widehat{v} \cos \varphi$,

i.e., the resulting Doppler shift $v_{\mathrm{D}}$ is proportional to the relative variability of the intensity fluctuations $\delta I / I=\widehat{I} / I_{0}$ and the velocity amplitude $\widehat{v}$. The intensity and velocity fluctuations $\widehat{I}$ and $\widehat{v}$ in Eq. (8) causing the net line shift $v_{\mathrm{D}}$ are spatially or temporally not resolved!

Nevertheless one might argue that the amplitude of the fluctuations on shorter not resolved time scales are related to those on larger time scales: a higher variability at high frequencies will in general be related to stronger slow fluctuations. This

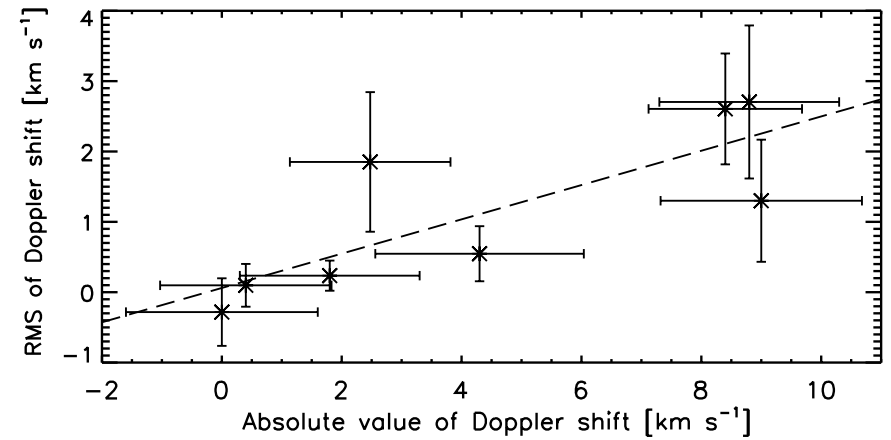

Fig. 3. RMS of Doppler shift vs. absolute mean Doppler shift. For the explanation of negative values see Sect. 3 .

speculation is based on the idea of turbulence, where the fluctuations on small and large scales are connected by a power law (e.g., Cally 1990 argued that turbulence plays an important role in the low transition region).

However, one has to be careful to simply copy the arguments of turbulence to the present situation. Dere (1989) analysed the power spectrum of spatial velocity fluctuations for the $1548 \AA$ and $1550 \AA$ lines of C IV formed at about $10^{5} \mathrm{~K}$. He found the power spectrum to be too flat to be due to a turbulent cascade, implying that most of the power is concentrated at small scales, and he speculated that some driving mechanism such as magnetic reconnection is maintaining the flat power spectrum. At other temperatures the shape of the power spectrum is not known.

In conclusion it seems plausible that within the framework of this simplistic model one might relate the un-resolved fluctuations $\widehat{I}$ and $\widehat{v}$ in Eq. (8) to the observed temporally and spatially resolved (RMS) fluctuations $I_{\mathrm{RMS}}$ and $v_{\mathrm{RMS}}$. Then from Eq. (8) it follows that $v_{\mathrm{D}} \propto I_{\mathrm{RMS}} / I_{0}$, which is in good agreement with the linear relation between the observed Doppler shifts and intensity (RMS) fluctuations shown in Fig. 1d.

Following the suggestion of Peter \& Judge (1999) below $4 \times 10^{5} \mathrm{~K}$ the redshifts are caused by downward propagating waves, above that temperature the blueshifts are caused by upward propagating waves: the phase lag $\varphi$ has a jump from 0 to $\pi$, i.e., $\cos \varphi$ changes sign there. Thus for the absolute value of the Doppler shift one expects $\left|v_{\mathrm{D}}\right| \propto v_{\mathrm{RMS}}$. The observed relation between Doppler shift and Doppler shift (RMS) variations is not as clear as for $v_{\mathrm{D}} \propto I_{\mathrm{RMS}}$ (a correlation coefficient of 0.58 compared to 0.92), but still remarkable (Fig. 3).

Of course, this simple model can only outline an interpretation of the relations of the observed RMS fluctuations to the Doppler shift, but it suggests that the transition region line shifts are a consequence of compressible fluctuations. A more detailed (numerical) modeling for a "realistic" transition region is needed for further insight. Such work is in progress.

\subsection{Connection between the transition region and the corona}

There is a long-standing debate whether or not there is a continuous connection from the chromosphere to the corona. "Classical" transition region models like that of Gabriel (1976) assume that the transition region emission originates from the 
thermal interface between the chromosphere and the corona. Here, transition region emission stands for lines formed at temperatures from say $20000 \mathrm{~K}$ to below $10^{6} \mathrm{~K}$, if one assumes ionisation equilibrium. "Non-classical" transition region models propose that this emission comes from a structure or structures not connected to the corona (e.g., Feldman 1983, 1998). However, it is not possible to decide between these two radically different pictures based on observations alone, but one has to make implicit assumptions on the physical nature of the structure in question (Judge \& McIntosh 1999). For example, Wikstøl et al. (1998) showed that most of the "observational facts" usually used to argue in favour of a "non-classical" transition region can also be reproduced in a forward model of a "classical" continuous transition region from the chromosphere to the corona when accounting for the dynamic nature of this region. This whole debate is thoroughly reviewed by Judge \& McIntosh (1999).

The results of the present study as shown in Figs. 1-3 add a valuable piece of information to this discussion. When plotting emission measures or Doppler shifts versus formation temperature (Figs. 2a and 1c) the lines fall into two groups with formation temperatures below and above $\sim 3 \times 10^{5} \mathrm{~K}$. This is not the case for the "scatter plots" of intensity fluctuation vs. Doppler shift or DEM (Figs. 1d and 2b) as well as for Doppler shift fuctuations vs. Doppler shift (Fig. 3). For a "non-classical" transition region model this suggests that in the different (geometrically not connected) structures the same process is producing the line shifts.

\section{Conclusions}

We have investigated the variation of brightness and Doppler shifts from the quiet Sun using time series obtained by SUMER and CDS in chromospheric, transition region and coronal lines. We found a high correlation of 0.92 between averaged Doppler shifts and relative intensity variabilities of the lines studied. The fact that the relation between these two quantities is the same for transition region and coronal lines argues that the same physical process is acting to produce the net Doppler shift, intensity variability and probably Doppler shift variability in the different atmospheric layers.

Based on the data for this analysis we found a less significant correlation between the intensity variability and the differential emission measure (correlation coefficient -0.78), which indicates that simple selection effects are at most a part of the reason for the correlation between intensity variability and Doppler shift.

Assuming that the connection between the net Doppler shifts and the relative RMS intensity fluctuations is based on compressible fluctuations like sound waves we show that a simple analytical model can nicely reproduce the statistical relations between the Doppler shifts, Doppler shift variations and intensity variations. However, further modelling, that is beyond the scope of this work, is needed to provide a more solid foundation to these interpretations.

Acknowledgements. We are grateful to the SUMER and CDS teams and their open data policy. The SUMER project is financially supported by DLR, CNES, NASA and the ESA PRODEX programme (Swiss contribution). We thank the referee Philip Judge for carefully reading and thoroughly commenting on the manuscript. AB thanks J. O. Stenflo and M. C. E. Huber for their encouragement and support. The work of AB was supported by the Swiss National Science Foundation grant No. 21-45083.95, by a grant from the ETH-Zürich, and by the Deutsche Forschungsgemeinschaft grant No. PE 782, which is gratefully acknowledged. SOHO is a mission of international cooperation between ESA and NASA.

\section{References}

Antiochos, S. K. 1984, ApJ, 280, 416

Athay, R. G., Gurman, J. B., Henze, W., \& Shine, R. A. 1983, ApJ, 265,519

Brekke, P., Hassler, D. M., \& Wilhelm, K. 1997, Sol. Phys., 175, 349

Brković, A., Rüedi, I., Solanki, S. K., et al. 2000, A\&A, 353, 1083

Brković, A., Solanki, S. K., \& Rüedi, I. 2002, A\&A, 385, 257

Cally, P. S. 1990, ApJ, 355, 693

Dere, K. P. 1989, ApJ, 340, 599

Dere, K. P., Bartoe, J.-D. F., \& Brueckner, G. E. 1984, ApJ, 281, 870

Dere, K. P., Landi, E., Mason, H. E., Monsignori Fossi, B. C., \& Young, P. R. 1997, A\&AS, 125, 149

Feldman, U. 1983, ApJ, 275, 367

Feldman, U. 1998, ApJ, 507, 974

Gabriel, A. 1976, Phil. Trans. Royal Soc. London, 281, 339

Gebbie, K. B., Hill, F., November, J., et al. 1981, ApJ, 251, L115

Hansteen, V. H. 1993, A\&A, 402, 741

Hansteen, V. H., Betta, R., \& Carlsson, M. 2000, A\&A, 360, 742

Harrison, R. A. 1997, Sol. Phys., 175, 467

Harrison, R. A., Sawyer, E. C., Carter, M. K., et al. 1995, Sol. Phys., 162,233

Judge, P. G., \& McIntosh, S. W. 1999, Sol. Phys., 190, 331

Krucker, S., Benz, A. O., Bastian, T. S., \& Acton L. W. 1997, ApJ, 488, 499

Landini, M., \& Monsignori Fossi, B. C. 1990, A\&AS, 82, 229

Mariska, J. T. 1988, ApJ, 334, 489

Mariska, J. T. 1992, The Solar Transition Region (Cambridge Univ. Press)

McClymont, A. N., \& Craig, I. J. D. 1987, ApJ, 312, 402

Peter, H., \& Judge, P. G. 1999, ApJ, 522, 1148

Pauluhn, A., Solanki, S. K., Rüedi, I., Landi, E., \& Schühle, U. 2000, A\&A, 362, 737

Rabin, D., \& Dowdy, J. F. Jr. 1992, ApJ, 398, 665

Spadaro, D., Lanza, A. F., \& Antiochos, S. K. 1996, ApJ, 462, 1011

Stucki, K., Solanki, S. K., Schühle, U., \& Rüedi, I. 2000, A\&A, 362, L49

Teriaca, L., Banerjee, D., \& Doyle, J. G. 1999, A\&A, 349, 636

Wikstøl, Ø., Judge, P. J., \& Hansteen, V. 1997, ApJ, 483, 972

Wikstøl, Ø., Judge, P. J., \& Hansteen, V. 1998, ApJ, 501, 895

Wilhelm, K., Curdt, W., Marsch, E., et al. 1995, Sol. Phys., 162, 189

Wilhelm, K., Lemaire, P., Dammasch, I. E., et al. 1998, A\&A, 334, 685 